J Neurosurg Case Lessons 1(6):CASE20112, 2021

DOI: $10.3171 /$ CASE20112

\title{
Cerebellar high-grade glioma with H3 K27M mutation: illustrative case
}

\author{
Mikiko Takahashi, MD, PhD, ${ }^{1}$ Yusuke Tomita, MD, ${ }^{2}$ Shoko Merrit Yamada, MD, PhD, ${ }^{2}$ Kazuto Yamazaki, MD, PhD, ${ }^{3}$ Hideaki Yokoo, MD, PhD, ${ }^{4}$ \\ Tatsuya Aso, MD, PhD, ${ }^{1}$ and Masashi Kawamoto, MD, PhD ${ }^{1}$
}

Departments of ${ }^{1}$ Diagnostic Pathology and ${ }^{2}$ Neurosurgery, Teikyo University Hospital, Mizonokuchi, Kawasaki, Kanagawa, Japan; ${ }^{3}$ Department of Pathology, Teikyo University Chiba Medical Center, Ichihara, Chiba, Japan; and `Department of Human Pathology, Gunma University Graduate School of Medicine, Maebashi, Gunma, Japan

\begin{abstract}
BACKGROUND Diffuse midline glioma H3 K27M-mutant is a distinct subtype of glial tumors newly introduced in the revised fourth edition of the World Health Organization Classification of Tumours of the Central Nervous System. They are aggressive pediatric tumors with a poor prognosis but have also been reported in adults.

OBSERVATIONS The authors present the case of a man in his $60 \mathrm{~s}$ who presented with rotatory vertigo, taste disorder, and right facial paralysis. Magnetic resonance imaging showed a tumor expanding from the cerebellum to the pons, which was histologically identified as glioblastoma, grade IV, IDH wild type. After tumor resection, the patient received chemoradiotherapy but showed only a partial response. His condition gradually worsened, and he died of progressive disease 12 months postoperation, after which an autopsy was performed. Tumor cells with a high nuclear-to-cytoplasm ratio were immunohistochemically analyzed and found to test positive for $\mathrm{H} 3 \mathrm{~K} 27 \mathrm{M}$ and negative for $\mathrm{H} 3 \mathrm{~K} 27 \mathrm{me}$. Furthermore, mutational analysis revealed HIST1H3B K27M mutation, and the tumor was finally identified as a high-grade glioma H3 K27M-mutant. The tumor invaded widely along the cerebral ventricle and disseminated to the spinal cord.
\end{abstract}

LESSONS When a glioblastoma shows localization or dissemination patterns different from those of typical glioblastoma, an $\mathrm{H} 3 \mathrm{~K} 27 \mathrm{M}-\mathrm{mutant}$ glioma should be suspected.

https://thejns.org/doi/abs/10.3171/CASE20112

KEYWORDS high-grade glioma; H3 K27M mutation; adult; cerebellum; dissemination

A diffuse midline glioma $\mathrm{H} 3 \mathrm{~K} 27 \mathrm{M}$-mutant, introduced in the revised fourth edition of the 2016 World Health Organization (WHO) Classification of Tumours of the Central Nervous System, is classified as a grade IV high-grade glioma. ${ }^{1}$ It commonly emerges in the brainstem, thalamus, and spinal cord and is associated with a poor prognosis. Although it is predominant in children, it has also been reported to occur in adults. ${ }^{2,3}$ We report an autopsy case of a high-grade glioma $\mathrm{H} 3$ $\mathrm{K} 27 \mathrm{M}$-mutant in an adult male patient.

\section{Illustrative Case}

A male in his 60 s presented to our hospital with rotatory vertigo, a taste disorder, and right facial paralysis. Magnetic resonance imaging (MRI) of the brain revealed a tumor distributed from the right pontomedullary junction to the inferior cerebellar peduncle (Fig. 1). During surgery, the tumor was exposed on the surface with adhesion of the facial and vestibulocochlear nerve and was found to be protruding into
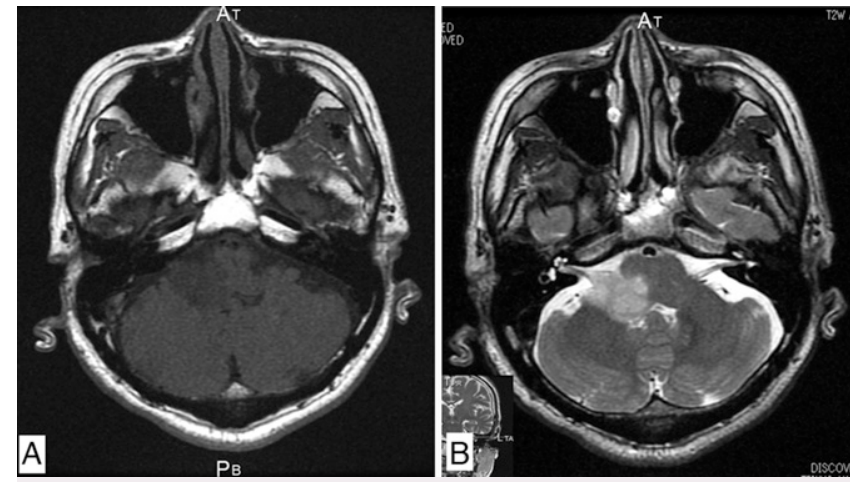

FIG. 1. Radiological features of the primary lesion at the time of surgery. MRI examination showed a tumor from the right pontomedullary junction to the inferior cerebellar peduncle with T1-weighted image (WI) hypointensity (A) and T2-WI hyperintensity (B).

ABBREVIATIONS MRI = magnetic resonance imaging; $W H O=$ World Health Organization .

INCLUDE WHEN CITING Published February 8, 2021; DOI: 10.3171/CASE20112.

SUBMITTED November 16, 2020. ACCEPTED December 7, 2020.

(c) 2021 The authors, CC BY-NC-ND 4.0 (http://creativecommons.org/licenses/by-nc-nd/4.0/). 

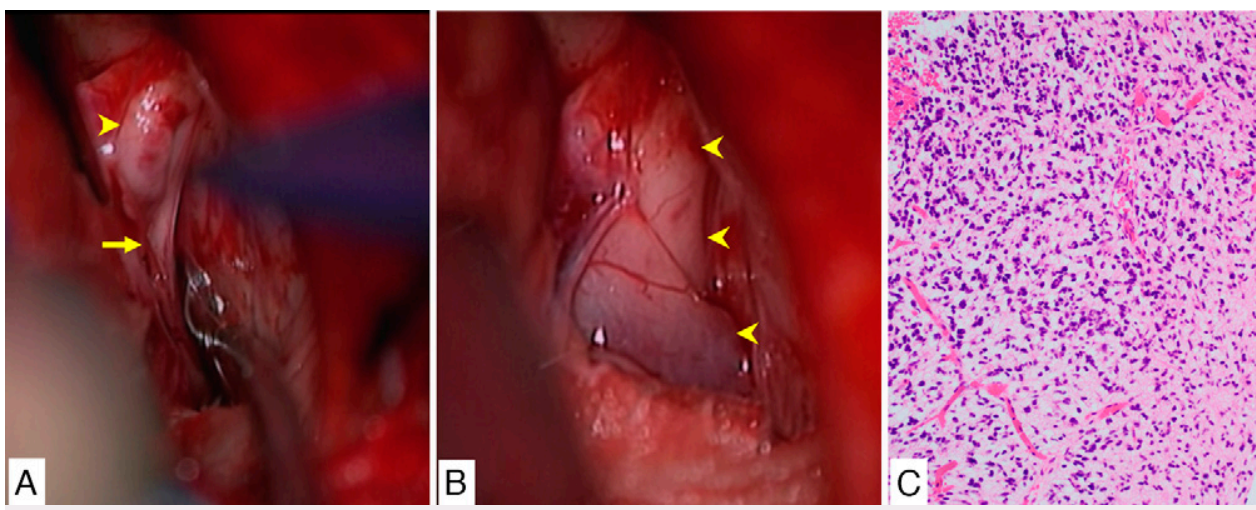

FIG. 2. Surgical and microscopic findings of the tumor. The tumor (arrowhead) adhered to the vestibulocochlear nerve (arrow) (A) and protruded into the pontocerebellar cistern (arrowheads) (B). C: Original magnification $\times 40$. Tumor cells with nuclei of various sizes distributed diffusely with proliferated microvessels.

the pontocerebellar cistern (Fig. 2A and B). Histological diagnosis of the tumor revealed a glioblastoma, grade IV, IDH wild type with hypercellularity and nuclei of various sizes (Fig. 2C). Tests for detection of the H3 K27M-mutant were not yet performed. Chemotherapy with temozolomide and bevacizumab and radiotherapy regimens were administered postsurgery. The patient initially showed a partial response to the treatment; however, the temozolomide was canceled given the emergence of myelosuppression 6 months later. Thereafter, his condition worsened and he was hospitalized; he later died of aspiration pneumonia, after which an autopsy was performed. Incidental findings from the patient's autopsy revealed that, macroscopically, the tumor involved the brainstem without a nodular growth pattern (Fig. 3A). It invaded along the cerebral ventricle and cistern with a thickness of several centimeters, consistent with the distribution pattern shown in his MRI examination, forming nodules in the ventricle (Fig. 3B and C). Additionally, the tumor disseminated to the spinal cord and formed nodules on the cauda equina (Fig. 3D). Microscopically, tumor cells with oval or short, rod-shaped nuclei were diffusely distributed at high cellularity and showed increased mitosis (Fig. 4A and $B$ ). They infiltrated around the blood vessels and peripheral nerves (Fig. 4C). Immunohistochemical analysis revealed that the tumor cells were partial positive for GFAP, S-100, nestin, and olig2; positive for $\mathrm{H} 3$

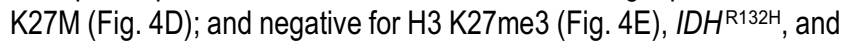
ATRX. Mutational analysis showed a K27M mutation in HIST1H3B (Fig. 4F). Finally, this tumor was identified as a cerebellar high-grade glioma H3 K27M-mutant.

\section{Discussion}

Diffuse midline glioma H3 K27M-mutant harbors heterozygous mutations in the H3F3A gene, encoding histone $\mathrm{H} 3.3$ or, in the $\mathrm{HIST} 1 \mathrm{H} 3 \mathrm{~B} / \mathrm{C}$ gene, encoding histone H3.1.1-3 Several studies have reported its occurrence in adults and at different anatomical localizations except the midline area. ${ }^{4-9}$ Additionally, the histological phenotypes of $\mathrm{H} 3 \mathrm{~K} 27 \mathrm{M}$-mutant gliomas have been reported to have various morphologies ranging from WHO grades I to IV. ${ }^{10,11}$
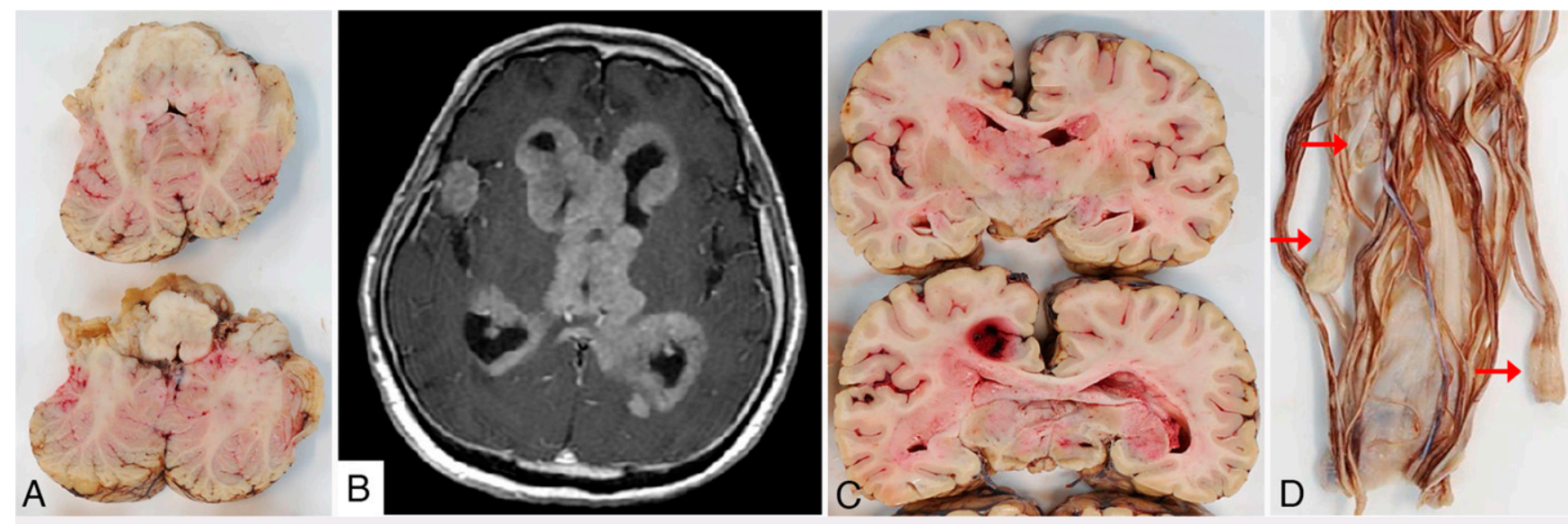

FIG. 3. Macroscopic and radiological features of the tumor. A: The tumor involved the brainstem without nodule formation. B: MRI examination at the time of the clinical exacerbation showed invasion along the cerebral ventricle with gadolinium-enhanced T2-WI hyperintensity. C: The tumor proliferated in the cerebral ventricle and formed the tumor surrounded with hemorrhage. D: The tumor disseminated to the spinal cord and formed nodules on the cauda equina (arrows). 

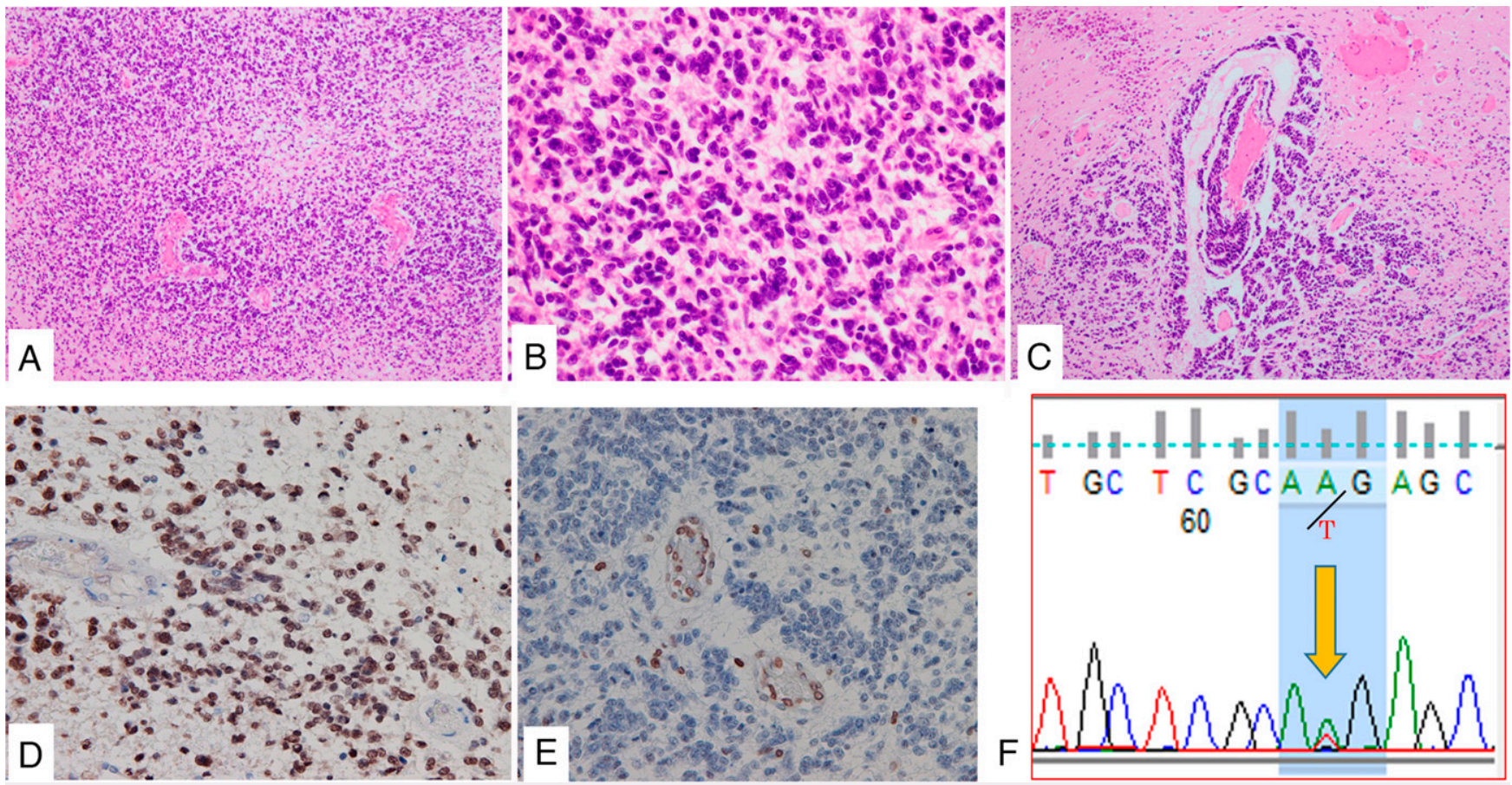

FIG. 4. Microscopic findings and mutational analysis of the autopsy specimen. A: Original magnification $\times 40$. The tumor cells in high cellularity distributed diffusely. B: Original magnification $\times 200$. The nuclei of the tumor cells were oval or short, rod-shaped forms and showed heavy signs of mitosis. C: Original magnification $\times 40$. The tumor cells infiltrated along the vessels, forming lines. Immunohistochemically, the tumor cells were positive for H3 K27M (D; original magnification $\times 200$ ) and negative for H3 K27me3 (E; original magnification $\times 200$ ). F: Sanger sequencing showed a lysine-to-methionine substitution at codon 27 (arrow).

\section{Observations}

In the present case, the tumor was initially identified as a glioblastoma, IDH wild type, but it was different from typical glioblastoma in the following ways: (1) it was located in the infratentorial area, (2) it protruded into the pontocerebellar cistern, and (3) it disseminated extensively without enlargement of the primary site. The characteristic infiltrative spread in glioblastomas is reportedly the rapid invasion of the neighboring brain structures. ${ }^{1}$ Autopsy-based analysis of diffuse intrinsic pontine glioma, a pediatric tumor integrated with diffuse midline glioma in the WHO 2016 classification, showed leptomeningeal dissemination in about $40 \%$ of the cases; ${ }^{12}$ however, such dissemination patterns are reported in only a few adult cases of $\mathrm{H} 3 \mathrm{~K} 27 \mathrm{M}$-mutant glioma. The pattern of spread in this case (infiltration along the cerebral ventricle with some thickness) may be one of the characteristics of an H3 K27M-mutant glioma.

\section{Lessons}

In conclusion, we reported an autopsy case of a cerebellar highgrade glioma H3 K27M-mutant. Diffuse midline glioma H3 K27Mmutant is predominant in children but also occurs in adults and at different anatomical localizations apart from the midline area. Its histological phenotype has been reported to have various morphologies. When a glioblastoma shows localization or dissemination patterns different from the typical glioblastoma, an H3 K27M-mutant glioma should be suspected.

\section{Acknowledgments}

We are grateful to Editage for editing our manuscript.

\section{References}

1. Hawkins CE, Ellison DW, Sturm D. Diffuse midline glioma, $H 3$ K27M-mutant. In: Louis DN, Ohgaki H, Wiestler OD, et al, eds. WHO Classification of Tumours of the Central Nervous System. JARC Press; 2016:57-59.

2. Wu G, Broniscer A, McEachron TA, et al. Somatic histone H3 alterations in pediatric diffuse intrinsic pontine gliomas and nonbrainstem glioblastomas. Nat Genet. 2012;44(3):251-253.

3. Wu G, Diaz AK, Paugh BS, et al. The genomic landscape of diffuse intrinsic pontine glioma and pediatric non-brainstem high-grade glioma. Nat Genet. 2014;46(5):444-450.

4. Meyronet D, Esteban-Mader M, Bonnet C, et al. Characteristics of H3 K27M-mutant gliomas in adults. Neuro Oncol. 2017;19(8): 1127-1134.

5. Yekula A, Gupta M, Coley N, et al. Adult H3K27M-mutant diffuse midline glioma with gliomatosis cerebri growth pattern: case report and review of the literature. Int J Surg Case Rep. 2020;68: 124-128.

6. Nakata S, Nobusawa S, Yamazaki T, et al. Histone H3 K27M mutations in adult cerebellar high-grade gliomas. Brain Tumor Pathol. 2017;34(3):113-119.

7. Funata N, Nobusawa S, Nakata S, et al. A case report of adult cerebellar high-grade glioma with $\mathrm{H} 3.1 \mathrm{~K} 27 \mathrm{M}$ mutation: a rare example of an H3 K27M mutant cerebellar tumor. Brain Tumor Pathol. 2018;35(1):29-35.

8. Lu VM, Akinduro OO, Daniels DJ. H3K27M mutation in adult cerebellar glioblastoma. J Clin Neurosci. 2020;71:316-317.

9. Wang L, Li Z, Zhang M, et al. H3 K27M-mutant diffuse midline gliomas in different anatomical locations. Hum Pathol. 2018;78: 89-96. 
10. Kleinschmidt-DeMasters BK, Mulcahy Levy JM. H3 K27M-mutant gliomas in adults vs. children share similar histological features and adverse prognosis. Clin Neuropathol. 2018;37(2):53-63.

11. Ebrahimi A, Skardelly M, Schuhmann MU, et al. High frequency of H3 K27M mutations in adult midline gliomas. J Cancer Res Clin Oncol. 2019;145(4):839-850.

12. Buczkowicz $P$, Bartels U, Bouffet $E$, et al. Histopathological spectrum of paediatric diffuse intrinsic pontine glioma: diagnostic and therapeutic implications. Acta Neuropathol. 2014;128(4): 573-581.

\section{Disclosures}

The authors report no conflict of interest concerning the materials or methods used in this study or the findings specified in this paper.

\section{Author Contributions}

Conception and design: Takahashi, Yokoo, Aso. Acquisition of data: Takahashi, Tomita, Yamazaki, Yokoo. Analysis and interpretation of data: Takahashi, Yamada, Yamazaki, Yokoo, Kawamoto. Drafting the article: Takahashi, Yokoo. Critically revising the article: Tomita, Yokoo. Reviewed submitted version of manuscript: Takahashi, Tomita, Yamada, Yokoo, Kawamoto. Approved the final version of the manuscript on behalf of all authors: Takahashi. Statistical analysis: Tomita. Administrative/technical/material support: Yokoo. Study supervision: Yokoo, Kawamoto.

\section{Correspondence}

Mikiko Takahashi: Teikyo University Hospital Mizonokuchi, Kanagawa, Japan. mikiko.ono@nifty.com. 